LCLS-TN-07-7

\title{
LCLS Undulator Quadrupole Fiducialization Plan
}

\author{
Zachary Wolf, Michael Levashov, \\ Eric Lundahl, Ed Reese, Catherine LeCocq, Robert Ruland \\ Stanford Linear Accelerator Center
}

August 14, 2007

\begin{abstract}
This note presents the fiducialization plan for the LCLS undulator quadrupoles. The note begins by summarizing the requirements for the fiducialization. A discussion of the measurement equipment is presented, followed by the methods used to perform the fiducialization and check the results. This is followed by the detailed fiducialization plan in which each step is enumerated. Finally, the measurement results and data storage formats are presented.
\end{abstract}

\section{Introduction $^{1}$}

The LCLS is made up of 33 assemblies consisting of an undulator, quadrupole, beam finder wire, and other components mounted on a girder. The components must be mounted in such a way that the beam passes down the axis of each component. In this note, we describe how the ideal beam axis is related to tooling balls on the quadrupole. This step, called fiducialization, is necessary because the ideal beam axis is determined magnetically, whereas tangible objects must be used to locate the quadrupole.

The note begins with the list of fiducialization requirements. The laboratory in which the work will be performed and the relevant equipment is then briefly described. This is followed by a discussion of the methods used to perform the fiducialization and the methods used to check the results. A detailed fiducialization plan is presented in which all the steps of fiducialization are enumerated. A discussion of the resulting data files and directory structure concludes the note.

\section{Requirements}

The LCLS quadrupole fiducialization requirements come from an undulator Physics Requirements Document ${ }^{2}$. The list of fiducialization requirements is given below.

\footnotetext{
${ }^{1}$ Work supported in part by the DOE Contract DE-AC02-76SF00515. This work was performed in support of the LCLS project at SLAC.

${ }^{2}$ H. D. Nuhn et al., "General Undulator System Requirements", LCLS Physics Requirements Document $1.4-001$.
} 
1. The transverse position of a zero integrated field line through the quadrupole must be known to $25 \mu \mathrm{m}$ in both $x$ and $y$ relative to tooling balls on the quadrupole.

2. The longitudinal position of the magnetic center of the quadrupole must be known in $z$ relative to tooling balls to $0.5 \mathrm{~mm}$.

3. Tooling ball locations must be specified such that the roll of the quadrupole is less than 5 mrad.

4. Tooling ball locations must be specified such that the pitch and yaw of the quadrupole are each less than $10 \mathrm{mrad}$.

\section{Equipment}

The quadrupoles will be fiducialized in the Magnetic Measurement Facility at SLAC. A vibrating wire system set up on a coordinate measuring machine (CMM) will be used to locate the transverse position of the ideal beam axis relative to the quadrupole tooling balls. The CMM will measure the roll and longitudinal position of the quadrupole relative to tooling balls. Optical alignment equipment will be used to check the fiducialization on the first few magnets.

The equipment used for the quadrupole fiducialization is illustrated in figure 1 . The

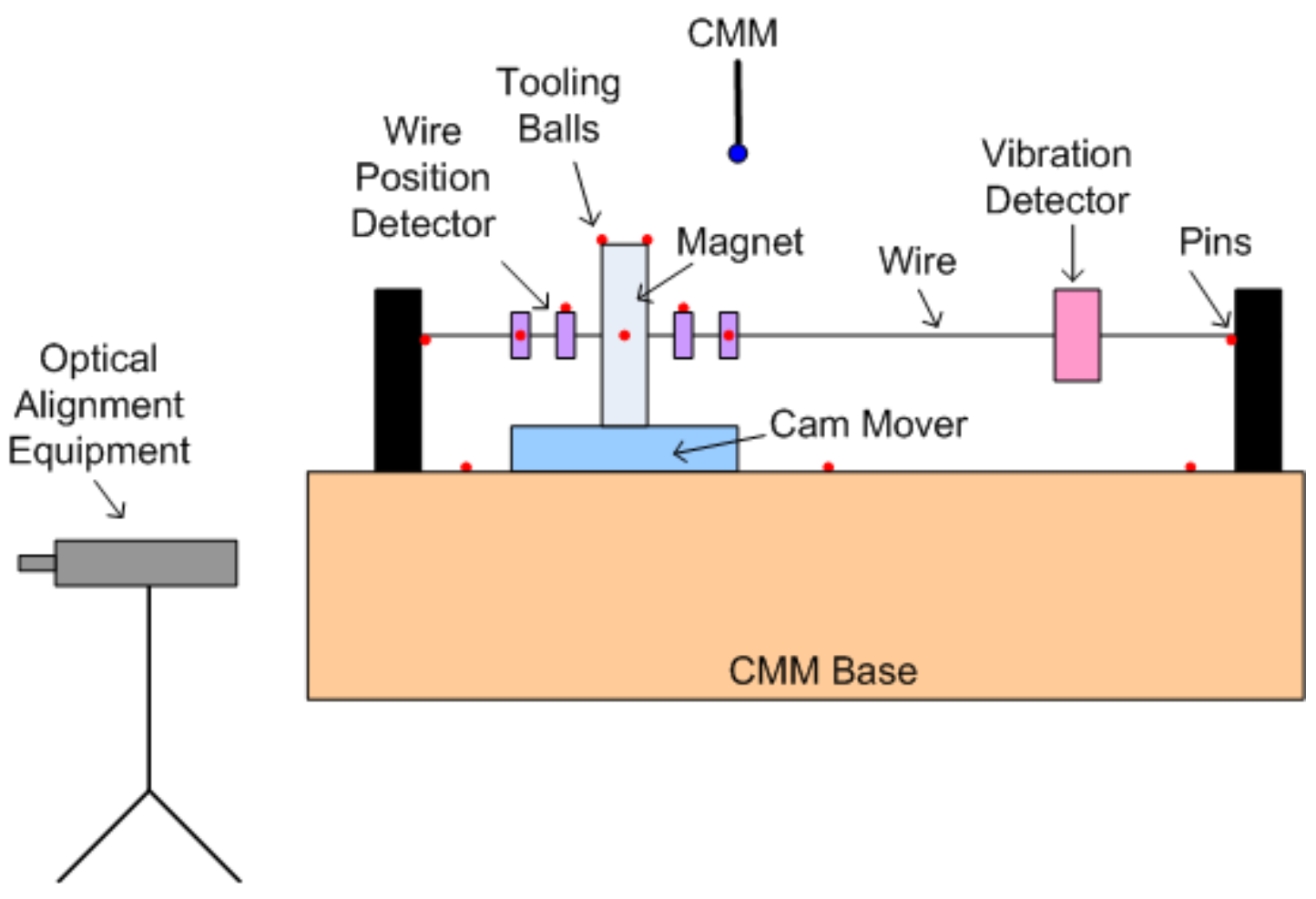

Figure 1: Equipment used for the quadrupole fiducialization. 
vibrating wire system uses a wire carrying alternating current going through the bore of the quadrupole. The frequency of the current in the wire is adjusted to a resonant frequency of vibration of the wire. When the wire is in the magnetic field of the quadrupole, the current in the wire interacts with the magnetic field and exerts a force on the wire at the resonant frequency of vibration, causing a large response. An optical sensor detects the wire vibration. When the quadrupole is moved so that the magnetic axis is along the wire, the magnetic field at the wire is zero and the vibration ceases. The $x, y$, pitch, and yaw of the quadrupole are determined. The fiducialization requirement is only that $x$ and $y$ be adjusted so that the integral of the field is zero. Adjusting pitch and yaw gives a unique fiducialization so the repeatability of the system can be determined. This is an extra feature of the method. The details of how the vibrating wire system works have been documented in a previous note ${ }^{3}$.

The quadrupole is placed on a cam mover system which also allows manual positioning. The operator moves the quadrupole by hand until the wire stops vibrating. At this point a computer program is run which moves the quadrupole with the cam movers. The program records the wire vibration amplitude and phase as a function of quadrupole position. The program calculates the zero vibration position and moves the quadrupole there. A useful check is to compare the position that the computer found to the position the operator found.

To fiducialize the quadrupole, the location of the wire must be determined relative to tooling balls on the quadrupole. A method must be used to locate the wire position which does not contact the wire. A set of wire position detectors, each consisting of a laser and a photodiode, is moved until the shadow of the wire is centered on each photodiode. The detector position where the wire shadow is centered on the photodiode is fixed relative to the tooling balls on the detector. The distance from this position to the tooling balls is determined by a calibration procedure. If the tooling ball location is measured, the position of the wire is known. The procedure for locating the wire, along with a description of calibrations and performance results has been given in a previous note ${ }^{4}$.

All tooling ball positions are measured with the CMM. This allows calculation of the wire position relative to tooling balls on the magnet. The $x$ and $y$ positions and the pitch and yaw of the magnetic center line relative to the quadrupole tooling balls are thus determined.

The CMM is also used to determine the quadrupole roll and $z$ position relative to the tooling balls. Measurements along the magnet poles are used to determine the roll. Measurements along the ends of the quadrupole are used to determine the $z$ position of the center of the quadrupole.

\section{Method}

This section presents details of the method used to carry out the fiducialization process. A naming convention is established and a coordinate system is defined. All measurement results will be presented using these names and this coordinate system. The process of fiducialization is presented. The magnet $z$ position and roll are measured and set

\footnotetext{
${ }^{3}$ Z. Wolf, "A Vibrating Wire System For Quadrupole Fiducialization", LCLS-TN-05-11, May, 2005.

${ }^{4}$ M. Levashov, Z. Wolf, "Set Up And Test Results For A Vibrating Wire System For Quadrupole Fiducialization", LCLS-TN-06-14, August, 2006.
} 
mechanically. The magnet is moved in $x, y$, pitch, and yaw until its center is aligned to the wire. The wire position is determined. All alignment quantities are related to tooling ball positions. The details of these steps will now be presented.

\subsection{Naming Convention}

Our naming convention is illustrated in figure 2. Similar components are named by a

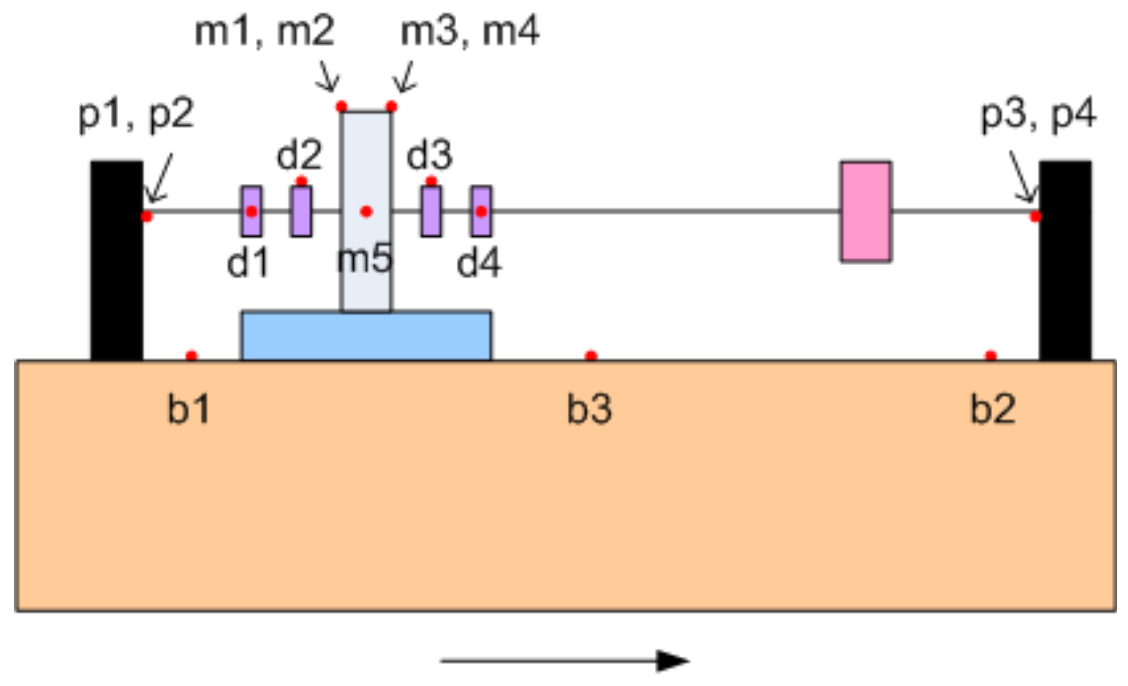

Beam Direction

Figure 2: The labels shown are used to identify components whose position must be determined.

unique letter followed by a sequential number. Numbering schemes in general progress from the magnet side of the system to the vibration detector side. This is the same as the electron beam direction through the magnet.

Pin $p 1$ positions the wire in $x, p 2$ in $y, p 3$ in $y$, and $p 4$ in $x$. This will be illustrated in more detail below. Wire position detector $d 1$ measures the $x$ position of the wire, $d 2$ the $y$ position, $d 3$ the $y$ position, and $d 4$ the $x$ position. The $y$ detectors are closest to the magnet to accurately account for wire sag. The tooling balls $m 1$ to $m 5$ are on the magnet. Their locations will be discussed further below. Tooling balls $b 1$ to $b 3$ are on the base of the system and are used to establish the coordinate system. $b 1$ and $b 2$ are along the wire, with $b 3$ off to the side.

\subsection{Coordinate System}

The coordinate system is illustrated in figure 3. The three tooling balls fixed to the support of the vibrating wire system are used to define a plane. An alignment crew will shim the tooling balls so the wire is parallel to the plane. The alignment crew will then shim the system support so the plane is perpendicular to gravity. This will simplify comparisons 


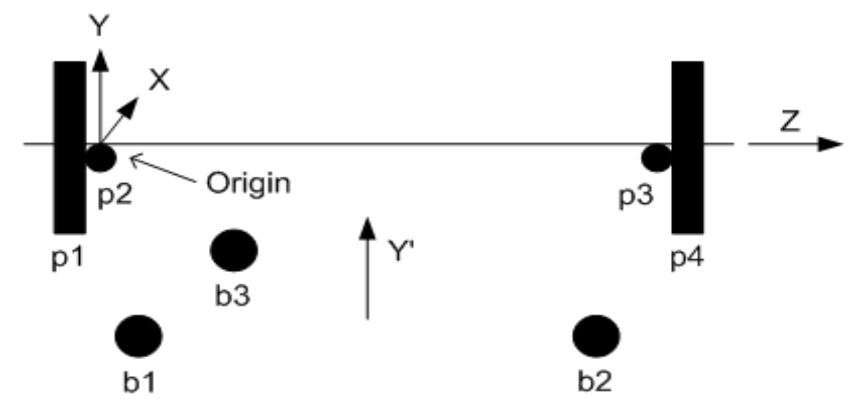

Figure 3: The coordinate system used in the quadrupole fiducialization has $z$ along the wire end points defined by pins $\mathrm{p} 1$ to $\mathrm{p} 4, y$ is up as defined by the normal to the plane of three tooling balls b1 to b3, and $x$ is horizontal such that it forms a right handed system.

between optical and CMM measurements. The normal to the plane, $y^{\prime}$ in the figure, will define the direction of the $y$ axis. The wire end points will define the $z$ axis. The wire is positioned by crossed pins. The CMM will scan the pins at each end of the wire and determine the center line of each pin. Knowing the pin diameters and the wire diameter, a point on each end of the wire is determined. The line between these points defines the $z$ axis. The $x$ axis is chosen perpendicular to $y$ and $z$ to form a right handed system. The origin is defined to be the last pin contact of the wire on the end of the wire closest to the magnet. This specification uniquely defines the coordinate system used for CMM and optical tooling measurements.

The relation of the coordinate system to the magnet is shown in figure 4 . The beam
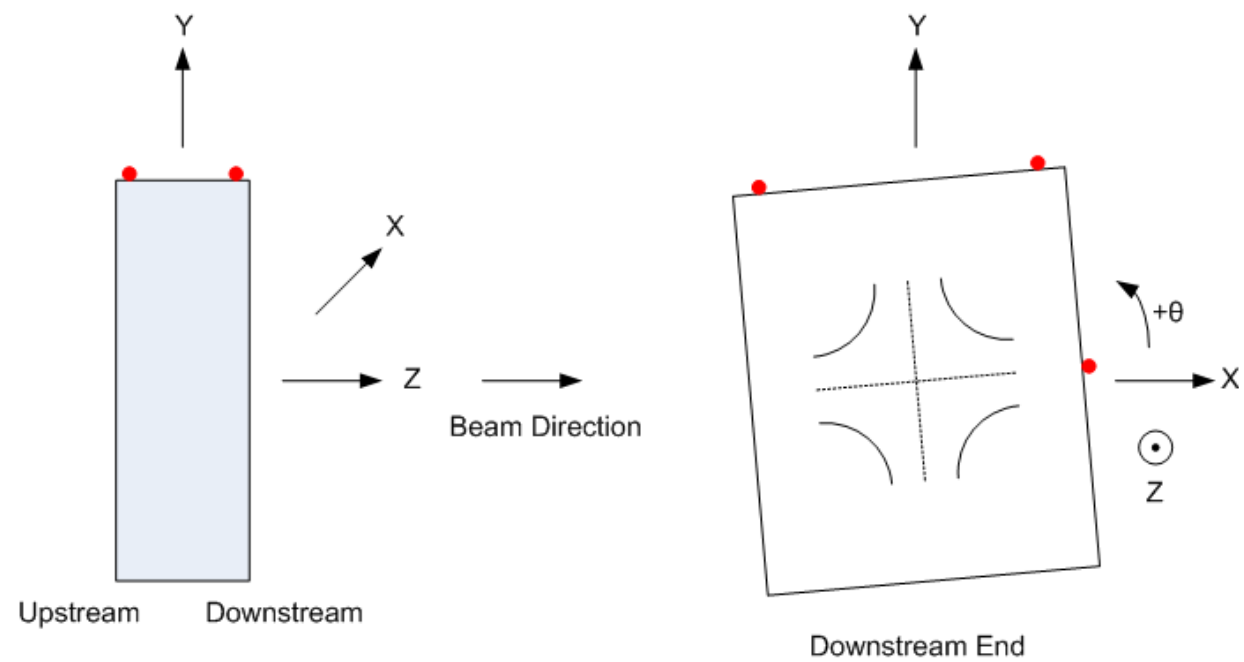

Figure 4: The orientation of the magnet in the coordinate system is illustrated.

moves from the upstream end of the magnet to the downstream end. The direction of the $z$ axis, from the magnet to the wire vibration detector, is chosen to coincide with the beam 
direction. Figure 4 illustrates the quadrupole with positive roll. Positive roll is defined as a positive rotation about the $z$ axis in the right handed sense. Positive pitch is defined as a negative rotation about the $x$ axis in the right handed sense. Positive yaw is defined as a positive rotation about the $y$ axis in the right handed sense.

\subsection{Procedure}

The steps to fiducialize the quadrupole proceed as follows. First, the quadrupole is placed on the stand and the roll and $z$ location are determined by the CMM. Adjustments to the roll are made until the roll is zero to within the specified limit. The $z$ location of the center of the quadrupole is determined by scans of the ends. The $z$ location is adjusted until it is at the first nodal position of the fourth vibration harmonic. A wire is then inserted into the quadrupole, tensioned, and alternating current at different resonant vibration frequencies is sent through the wire. The quadrupole is moved in $x, y$, pitch, and yaw until the wire vibration ceases. At this point, the quadrupole has the wire on the magnetic axis, which is the ideal beam axis. The wire position detectors are moved until they are centered on the wire. The CMM measures the coordinates of all tooling balls on both the magnet and the wire position detectors, and it locates the pins which position the wire as a check. The position of the wire relative to the magnet tooling balls is calculated.

\subsection{Wire Position}

The detectors used to determine the wire position and their relation to the magnet are illustrated in figure 5. The wire position detectors consist of a laser shining through a slit onto a detector. When this assembly is moved past the wire, the shadow of the wire causes the signal to decrease. The point where the wire shadow is centered on the slit is a fixed distance from tooling balls on the detector assembly. These distances are denoted by $c_{d 1}$, $c_{d 2}, c_{d 3}$, and $c_{d 4}$ in figure 5 . They are determined by a calibration procedure described in an LCLS Technical Note ${ }^{5}$.

After the magnet is moved so that the wire is along the magnetic axis, the wire position detectors are moved until their detection locations are centered on the wire. The CMM measures the coordinates of the center of the tooling balls denoted by $R=(x, y, z)$. In figure $5 R_{d 1}, R_{d 2}, R_{d 3}$, and $R_{d 4}$ are the positions of the $x$ and $y$ detector tooling balls. $R_{m a g}$ is a generic designation for the positions of the tooling balls on the magnet.

The position of the wire is calculated as follows. At the position of the upstream $x$ detector $z_{d 1}$, the $x$ position of the wire is $x_{d 1}-c_{d 1}$. Similarly, at the downstream detector at $z_{d 4}$, the $x$ position is $x_{d 4}-c_{d 4}$. The $x$ position of the wire at any $z$ is then given by

$$
x_{w}(z)=\left(x_{d 1}-c_{d 1}\right)+\frac{\left(x_{d 4}-c_{d 4}\right)-\left(x_{d 1}-c_{d 1}\right)}{\left(z_{d 4}-z_{d 1}\right)} \cdot\left(z-z_{d 1}\right)
$$

The $y$ position of the wire is assumed to vary linearly in the short distance between the position detectors. The equation for the $y$ position of the wire is given by

$$
y_{w}(z)=\left(y_{d 2}-c_{d 2}\right)+\frac{\left(y_{d 3}-c_{d 3}\right)-\left(y_{d 2}-c_{d 2}\right)}{\left(z_{d 3}-z_{d 2}\right)} \cdot\left(z-z_{d 2}\right)
$$

\footnotetext{
${ }^{5}$ M. Levashov, Z. Wolf, "Set Up And Test Results For A Vibrating Wire System For Quadrupole Fiducialization", LCLS-TN-06-14, August, 2006.
} 


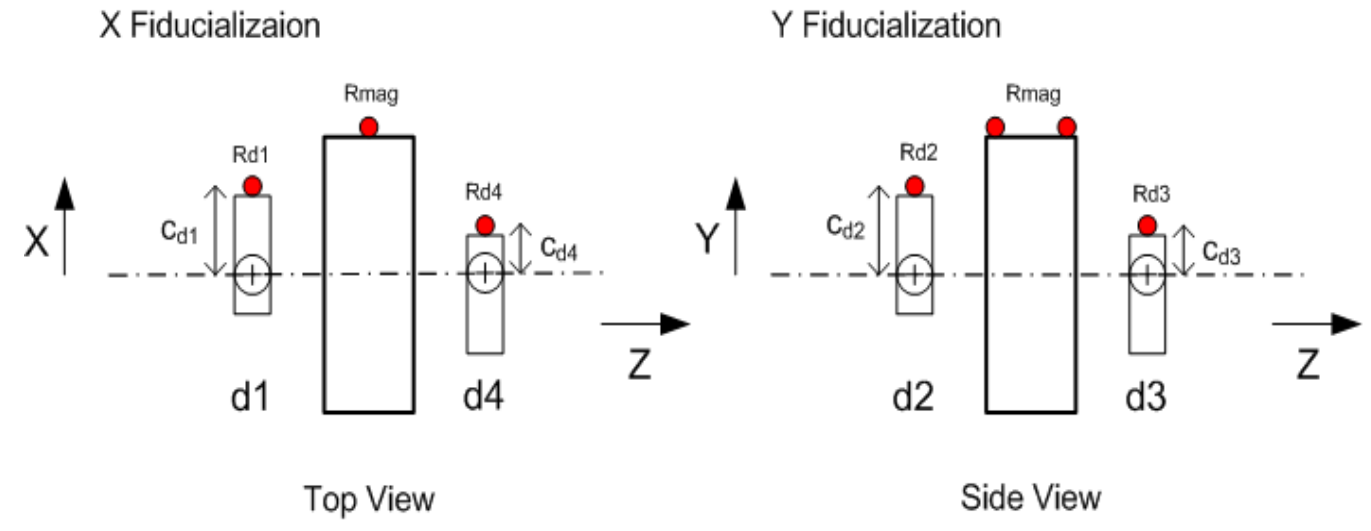

Figure 5: The wire position detector calibrations $\left(c_{d}\right)$ and the tooling ball positions for both the detectors $\left(R_{d}\right)$ and the magnet $\left(R_{m a g}\right)$ are required to fiducialize the quadrupole.

These equations will be used to calculate the magnet tooling ball locations relative to the wire.

\subsection{Quadrupole $z$}

The fiducialization of the quadrupole in $z$, as well as the fiducialization of the roll angle, will be done entirely on the CMM. Because of the loose tolerance on both the $z$ position and roll angle of the quadrupole, magnetic measurements are not required. The $z$ positions of the outside faces of the quadrupole will be measured by the CMM. The midpoint will be determined. The $z$ position of the midpoint will be related to the tooling balls. This is illustrated in figure 6 .

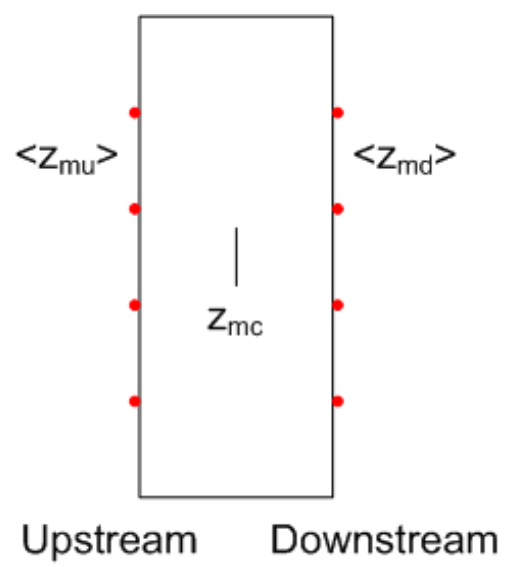

Figure 6: The $\mathrm{z}$ position of the center of the quadrupole is the average of the $\mathrm{z}$ positions of the magnet faces.

The CMM makes position measurements on the upstream face of the magnet. The 
average of the $z$ coordinates is $\left\langle z_{m u}\right\rangle$. Similarly, on the downstream face, the average of the $z$ coordinates is $\left\langle z_{m d}\right\rangle$. The $z$ position of the center is then

$$
z_{m c}=\frac{1}{2}\left(<z_{m u}>+<z_{m d}>\right)
$$

\subsection{Quadrupole Roll}

For roll, the pole shapes will be measured and the symmetry point on each pole will be determined. The angles of the lines between opposite symmetry points will be combined to determine the roll angle. This is illustrated in figure 7 .

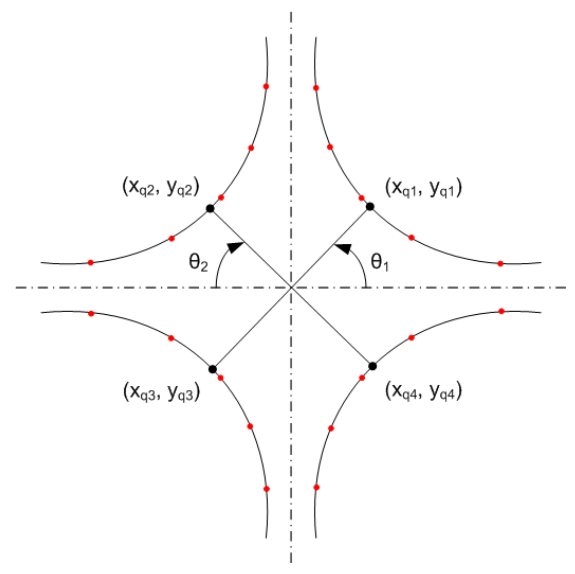

Figure 7: The roll of the quadrupole is determined by scanning the poles with the CMM.

Let the symmetry points of the four quadrants be at $\left(x_{q 1}, y_{q 1}\right), \ldots,\left(x_{q 4}, y_{q 4}\right)$. Opposite symmetry points are joined by line segments. The angles of the line segments are calculated as follows.

$$
\begin{gathered}
\theta_{1}=\tan ^{-1}\left(\frac{y_{q 1}-y_{q 3}}{x_{q 1}-x_{q 3}}\right) \\
\theta_{2}=\tan ^{-1}\left(-\frac{y_{q 2}-y_{q 4}}{x_{q 2}-x_{q 4}}\right)
\end{gathered}
$$

The roll of the quadrupole is defined as the angle of the poles relative to the $\pi / 4$ radian line. The angles $\theta_{1}$ and $\theta_{2}$ are related to the roll angle $\theta_{m}$ as follows.

$$
\begin{aligned}
& \theta_{1}=\frac{\pi}{4}+\theta_{m} \\
& \theta_{2}=\frac{\pi}{4}-\theta_{m}
\end{aligned}
$$

This gives

$$
\theta_{m}=\frac{1}{2}\left(\theta_{1}-\theta_{2}\right)
$$




\subsection{Quadrupole Tooling Balls}

The quadrupole has five tooling balls. A drawing ${ }^{6}$ of the quadrupole, with some additional annotations, is shown in figure 8 . In the figure, the tooling balls have been labeled $m 1$

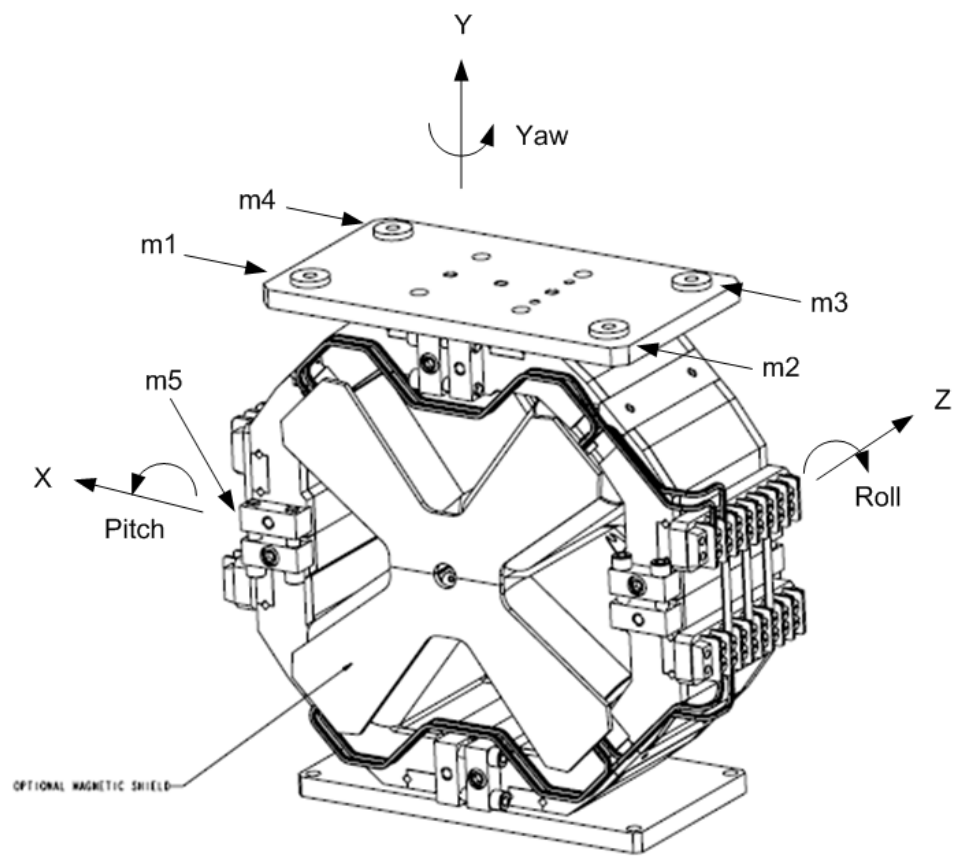

Figure 8: Drawing of the quadrupole showing the tooling ball locations.

through $m 5$. We denote the positions of the centers of the tooling balls on the magnet by $R_{m 1}$ to $R_{m 5}$. Individual coordinates are denoted by $x_{m 1}, y_{m 1}, z_{m 1}$, etc.

\subsection{Fiducialization}

To fiducialize the magnet, the tooling ball locations must be given such that the magnet's six degrees of freedom can be set to specified values. We must specify the $x$ and $y$ locations of the tooling balls relative to the wire, i.e. to the ideal beam axis. Also, the tooling ball positions relative to the magnet center in $z$ must be specified. We must specify the tooling ball positions when the magnet roll is zero, and when the magnetic axis' pitch and yaw relative to the wire are zero.

Tooling ball positions are found by the CMM. In many cases, it is useful to combine tooling ball positions to form quantities sensitive only to a single magnet coordinate. We find combinations of tooling balls which determine a single coordinate and are insensitive to errors in other coordinates. We describe the tooling ball combinations below. Using this information, the fiducialization parameters are calculated.

\footnotetext{
${ }^{6}$ SLAC Drawing Number SA-381-006-10, rev. 02.
} 
To find the $x$ position of the magnet relative to the wire, we use tooling ball 5 . Its position relative to the wire is given by

$$
x_{m}=x_{m 5}-x_{w}\left(z_{m 5}\right)
$$

where $x_{m 5}$ is the $x$ coordinate of tooling ball 5 and $x_{w}\left(z_{m 5}\right)$ is the $x$ coordinate of the wire at the $z$ position of tooling ball 5. $x_{m}$ is insensitive to the magnet $y$ and $z$ positions, and to magnet roll, pitch, and yaw. Errors in these quantities cause errors in $x_{m}$ which are at most second order.

To find the $y$ position of the magnet, we use a combination of the tooling balls on the top of the magnet. The combination is given as follows

$$
\begin{aligned}
& <y_{\text {top }}>=\frac{1}{4}\left(y_{m 1}+y_{m 2}+y_{m 3}+y_{m 4}\right) \\
& <z_{\text {top }}>=\frac{1}{4}\left(z_{m 1}+z_{m 2}+z_{m 3}+z_{m 4}\right)
\end{aligned}
$$

This average $y$ position is related to the wire position to give the $y$ fiducialization.

$$
y_{m}=<y_{\text {top }}>-y_{w}\left(<z_{\text {top }}>\right)
$$

where $\left.y_{w}\left(<z_{t o p}\right\rangle\right)$ is the $y$ position of the wire at $\left\langle z_{t o p}\right\rangle$. $y_{m}$ is insensitive to the $x$ and $z$ positions of the magnet, and to the magnet roll, pitch, and yaw. Errors in these quantities cause errors in $y_{m}$ which are second order or higher.

The $z$ position of the center of the magnet was given in equation 3. To fiducialize the magnet in $z$, we must relate this position to the tooling balls. We use $\left\langle z_{\text {top }}>\right.$ defined in equation 11 as the tooling ball $z$ position.

$$
z_{m}=z_{m c}-<z_{t o p}>
$$

$z_{m}$ is insensitive to the magnet $x$ and $y$ positions and to the magnet roll and yaw, with errors in these quantities causing second order or higher errors in $z_{m}$. There is, however, a first order error in pitch. The error on the pitch must be small enough so that the error on $z_{m}$ is within the specified limit.

The roll of the magnet is adjusted to be zero, within the specified limit. This angle must be reproduced when the magnet is used. The CMM determines the average angle of the poles $\theta_{m}$ with respect to the $x$ axis, as noted in equation 8 . Note that the $x$ axis must be related to gravity in order to use this information at a future date. $\theta_{m}$ must be zero within the specified limit on the roll angle. To fiducialize the roll of the magnet, form the following quantities.

$$
\begin{aligned}
y_{m 14} & =\frac{1}{2}\left(y_{m 1}+y_{m 4}\right) \\
x_{m 14} & =\frac{1}{2}\left(x_{m 1}+x_{m 4}\right) \\
y_{m 23} & =\frac{1}{2}\left(y_{m 2}+y_{m 3}\right) \\
x_{m 23} & =\frac{1}{2}\left(x_{m 2}+x_{m 3}\right)
\end{aligned}
$$


We define the roll of the magnet given by the tooling balls to be

$$
\operatorname{roll}_{m}=\tan ^{-1}\left(\frac{y_{m 14}-y_{m 23}}{x_{m 14}-x_{m 23}}\right)
$$

The roll is insensitive to errors in the magnet $x, y$, and $z$ positions, and to errors in the magnet pitch and yaw. (It is assumed that the tooling balls are symmetrically placed on the top of the magnet.)

The vibrating wire system is used to set the pitch of the magnet, the vertical angle of the magnet relative to the wire. Combinations of the tooling ball positions are used to reproduce this angle. Form the following quantities.

$$
\begin{aligned}
& y_{m 34}=\frac{1}{2}\left(y_{m 3}+y_{m 4}\right) \\
& z_{m 34}=\frac{1}{2}\left(z_{m 3}+z_{m 4}\right) \\
& y_{m 12}=\frac{1}{2}\left(y_{m 1}+y_{m 2}\right) \\
& z_{m 12}=\frac{1}{2}\left(z_{m 1}+z_{m 2}\right)
\end{aligned}
$$

The pitch is given by

$$
\text { pitch }_{m}=\tan ^{-1}\left(\frac{y_{m 34}-y_{m 12}}{z_{m 34}-z_{m 12}}\right)
$$

The pitch is insensitive to errors in the magnet $x, y$, and $z$ positions, and to errors in the magnet roll and yaw. Note that the definition of the $z$ axis did not include the sag of the wire. This introduces a very small error in the pitch.

The vibrating wire system is used to set the yaw of the magnet, the horizontal angle of the magnet relative to the wire. Combinations of the tooling ball positions are used to reproduce this angle. Form the following quantities.

$$
\begin{aligned}
x_{m 14} & =\frac{1}{2}\left(x_{m 1}+x_{m 4}\right) \\
z_{m 14} & =\frac{1}{2}\left(z_{m 1}+z_{m 4}\right) \\
x_{m 23} & =\frac{1}{2}\left(x_{m 2}+x_{m 3}\right) \\
z_{m 23} & =\frac{1}{2}\left(z_{m 2}+z_{m 3}\right)
\end{aligned}
$$

The yaw is given by

$$
y a w_{m}=\tan ^{-1}\left(\frac{z_{m 23}-z_{m 14}}{x_{m 14}-x_{m 23}}\right)
$$

The yaw is insensitive to errors in the magnet $x, y$, and $z$ positions, and to errors in the magnet roll and pitch. 


\section{Checks}

Several checks of the fiducialization have been implemented. They are discussed below.

\subsection{Computer Program Result Check}

The quadrupole mount is designed for both manual and automated movement. The operator first places the quadrupole on its mount and moves the quadrupole by hand until its magnetic axis is centered on the wire. The position that the operator leaves the quadrupole in defines the zero position for the automated motion system. The operator runs a computer program which moves the quadrupole in $x, y$, pitch, and yaw. The program determines the quadrupole position at which its magnetic axis is centered on the wire. This position should be close to the zero position. A deviation of more than a few microns in $x$ or $y$, or milliradians in pitch or yaw, indicates an error.

\subsection{Optical Alignment Check}

Optical alignment equipment will be used to check for errors in the $x$ and $y$ fiducialization. These quantities are critical and have tight tolerances. The accuracy of the optical measurements is roughly $50 \mu \mathrm{m}$. This check will find any large errors in the $x$ and $y$ fiducializations.

\subsection{1 $x$ Fiducialization}

To check the $x$ fiducialization, a jig transit is bucked into the pins which determine the $x$ position of the wire. This is illustrated in figure 9 . We use $h$ to represent horizontal measurements using the optical alignment equipment. Measurements $h_{p 1}$ and $h_{p 4}$ are

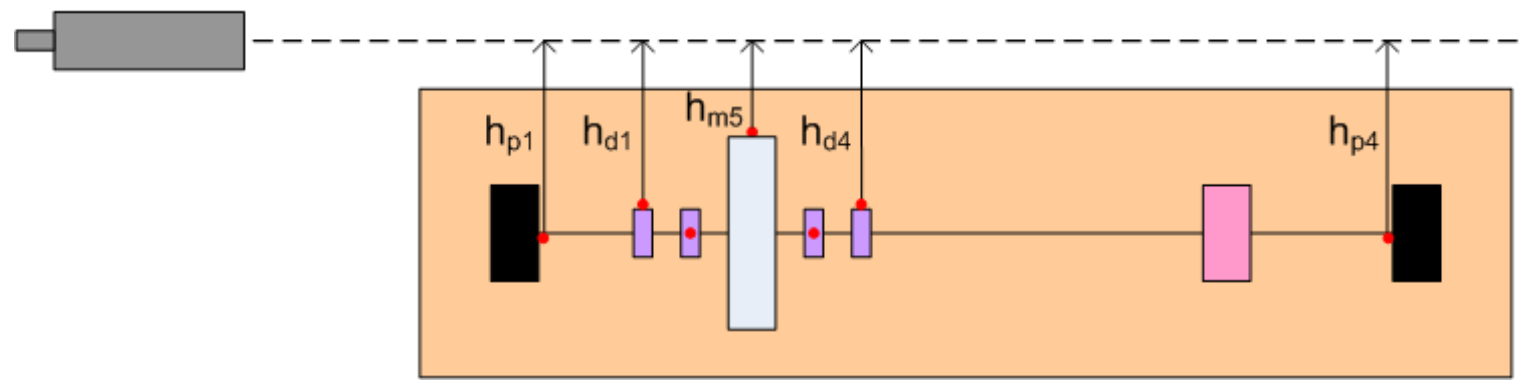

Figure 9: Optical alignment equipment is used to check the $x$ fiducialization.

made from the upstream and downstream pins, respectively, to the line of sight. The wire center is a wire radius $r_{w}$ back from the pin. Measurements $h_{d 1}$ and $h_{d 4}$ are made from the upstream and downstream wire position detectors, respectively, to the line of sight. Finally, measurement $h_{m 5}$ is made from tooling ball 5 on the magnet to the line of sight. The line of sight is parallel to the wire because of the procedure to buck in the jig transit.

The $x$ position of the wire relative to the line of sight is

$$
h_{w 1}=h_{p 1}-r_{w}
$$




$$
\begin{aligned}
& h_{w 2}=h_{p 4}-r_{w} \\
& h_{w 3}=h_{d 1}+c_{d 1} \\
& h_{w 4}=h_{d 4}+c_{d 4}
\end{aligned}
$$

These quantities must be equal.

$$
h_{w}=h_{w 1}=h_{w 2}=h_{w 3}=h_{w 4}
$$

If they are not, an error has occurred.

The relative distance between tooling ball 5 and the wire is

$$
h_{m}=h_{w}-h_{m 5}
$$

The optically determined quantity $h_{m}$ must agree with the $x$ fiducialization quantity $x_{m}$ or there is an error.

\subsection{2 $y$ Fiducialization}

To check the $y$ fiducialization, a sight level is used to measure vertical distances up to the level's line of sight as illustrated in figure 10 . We use $v$ to represent vertical measurements using the optical alignment equipment. The instrument resolution is $12.5 \mu \mathrm{m}$, its

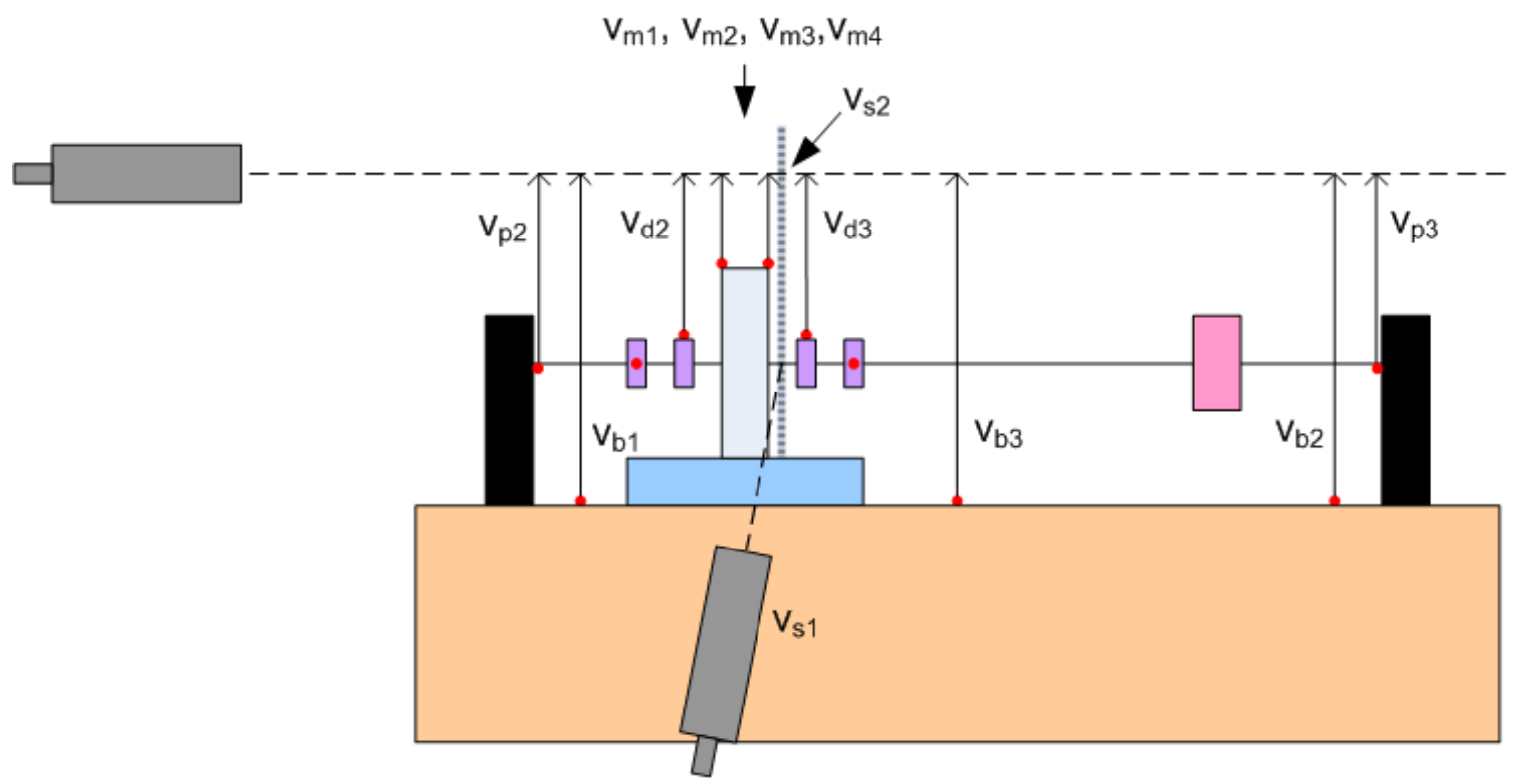

Figure 10: Optical measurements are used to check the $y$ fiducialization.

repeatability is estimated to be $25 \mu \mathrm{m}$ within the same setup and $50 \mu \mathrm{m}$ otherwise. The measurements $v_{p 2}$ and $v_{p 3}$ are to the upstream and downstream pins, respectively, which position the wire. The wire center is a wire radius $r_{w}$ above the pins. The measurements $v_{d 2}$ and $v_{d 3}$ are to the upstream and downstream wire position detectors, respectively. The 
measurements $v_{m 1}, v_{m 2}, v_{m 3}$ and $v_{m 4}$ are to the tooling balls on top of the magnet. The measurements $v_{b 1}, v_{b 2}$, and $v_{b 3}$ are to tooling balls fixed on the support of the vibrating wire system. They are also measured with the CMM and are used for relative position checks.

A second sight level is set up at the height of the wire and will look directly at the wire. Behind it, a ruler will be placed vertically with the help of the CMM. Both sight levels read the ruler, $v_{s 1}$ and $v_{s 2}$, to determine the distance from the wire to the upper sight level's line of sight.

As previously noted, an alignment crew initially shims the pins which support the wire vertically so that they are level. This is done in order to simplify the optical alignment checks. The sag of the wire is estimated to be $21 \mu \mathrm{m}^{7}$. This is within the uncertainty of this optical alignment check and will be neglected. For now, we will assume the wire is straight. The $y$ position of the wire relative to the line of sight is

$$
\begin{aligned}
& v_{w 1}=v_{p 2}-r_{w} \\
& v_{w 2}=v_{p 3}-r_{w} \\
& v_{w 3}=v_{d 2}+c_{d 2} \\
& v_{w 4}=v_{d 3}+c_{d 3}
\end{aligned}
$$

These quantities must be equal within the accuracy of the measurement.

$$
v_{w}=v_{w 1}=v_{w 2}=v_{w 3}=v_{w 4}
$$

If they are not, an error has occurred.

The second sight level provides a further check of the wire position relative to the upper level's line of sight. The upper level reads position $v_{s 2}$ on the vertical scale. The lower level, which is adjusted to be at the same height as the wire, reads $v_{s 1}$. The distance of the wire below the upper level's line of sight is

$$
v_{w s}=v_{s 1}-v_{s 2}
$$

Within measurement error, we must have

$$
v_{w}=v_{w s}
$$

Otherwise, an error has occurred.

The vertical distance from the upper level's line of sight to the tooling balls on the top of the magnet is given by $v_{m 1}, v_{m 2}, v_{m 3}$, and $v_{m 4}$. As during the fiducialization with the CMM, we form the quantity

$$
v_{m_{-} t b}=\frac{1}{4}\left(v_{m 1}+v_{m 2}+v_{m 3}+v_{m 4}\right)
$$

The relative vertical distance between the average of the $y$ tooling balls and the wire is

$$
v_{m}=v_{w}-v_{m-t b}
$$

The optically determined quantity $v_{m}$ must agree with the $y$ fiducialization quantity $y_{m}$ or there is an error.

\footnotetext{
${ }^{7}$ Z. Wolf, "A Vibrating Wire System For Quadrupole Fiducialization", LCLS-TN-05-11, May, 2005.
} 


\subsection{CMM Checks}

The CMM can do an accurate check that the wire was located properly. The support points of the wire are known, as are the location of the wire position detectors. The position of the wire at the detector locations can be calculated from the pin positions and the sag. The estimated accuracy of the CMM measurements is $5 \mu \mathrm{m}$ over its measurement volume. The calculated wire positions must agree with the measured positions within this accuracy.

The sag of the wire due to gravity, $y_{g}$, is given by the following formula ${ }^{8}$

$$
y_{g}(z)=\frac{m_{l} g}{2 T} z(z-L)
$$

where $m_{l}$ is the mass per unit length of the wire, $g$ is the gravitational acceleration, $T$ is the wire tension, and $L$ is the wire length. The CMM measures the $z$ positions of the wire position detectors. The measured wire position must agree with the $y$ value calculated from this formula.

The wire should be positioned repeatably by the pins. A further check is to compare the tooling ball positions of the wire position detectors from run to run. They should be the same at the few micron level.

\section{Fiducialization Plan}

\section{Preliminaries}

Commission the system as described in a previous note ${ }^{9}$. Calibrate the wire position detectors. Set up the vibrating wire system so the plane of the three tooling balls on the support is parallel to the wire. Shim the support so that the three tooling balls are all at the same height. Choose a reference magnet to be measured once per week during the production run.

2. Mechanically align the quadrupole to the test stand

(a) Level the quadrupole support.

(b) Place the quadrupole on the support.

(c) Determine the quadrupole center $z$ location with the CMM.

(d) Move the quadrupole until the center $z$ location is $1 / 4$ of the way from the upstream pin to the downstream pin.

(e) Determine the quadrupole roll angle with the CMM.

(f) Adjust the roll angle to be within $0.1 \mathrm{mrad}$ of zero.

3. Perform manual vibrating wire measurements

\footnotetext{
${ }^{8}$ Z. Wolf, "A Vibrating Wire System For Quadrupole Fiducialization", LCLS-TN-05-11, May, 2005.

${ }^{9}$ M. Levashov, Z. Wolf, "Set Up And Test Results For A Vibrating Wire System For Quadrupole Fiducialization", LCLS-TN-06-14, August, 2006.
} 
(a) String a wire through the quadrupole. Apply alternating current to the wire. Adjust the frequency of the alternating current to be at the 2'nd harmonic resonant frequency of vibration of the wire.

(b) Manually adjust the quadrupole mount in $x, y$ until the quadrupole axis is along the wire.

(c) Set the resonant frequency to the fourth harmonic.

(d) Adjust the pitch and yaw of the quadrupole until the wire stops vibrating.

(e) Iterate the above steps until the quadrupole is aligned to the wire in $x, y$, pitch, and yaw.

(f) Leave the magnet in this position. It defines the zero position for the measurement program.

4. Perform automated vibrating wire measurements

(a) Run the automated measurement program.

(b) The program leaves the quadrupole axis aligned to the wire. Check that this position is the zero position defined in the preceding step.

5. Perform CMM measurements

(a) Locate the pins which position the wire with the CMM. These define the $z$ axis. (The three tooling balls located in the next step may eventually be used to define the $z$ axis once offsets to the pins are determined.)

(b) Locate the three tooling balls on the support of the vibrating wire system which define the direction of the $y$ axis.

(c) Establish the coordinate system for the measurements.

(d) Measure all tooling ball locations.

6. Perform optical alignment checks

(a) Buck in the instruments.

(b) Measure all tooling ball locations, pin locations, and scale locations.

7. Analyze the results

(a) Calculate the fiducialization results according to the formulas given in this note.

(b) Perform all checks described in this note. 


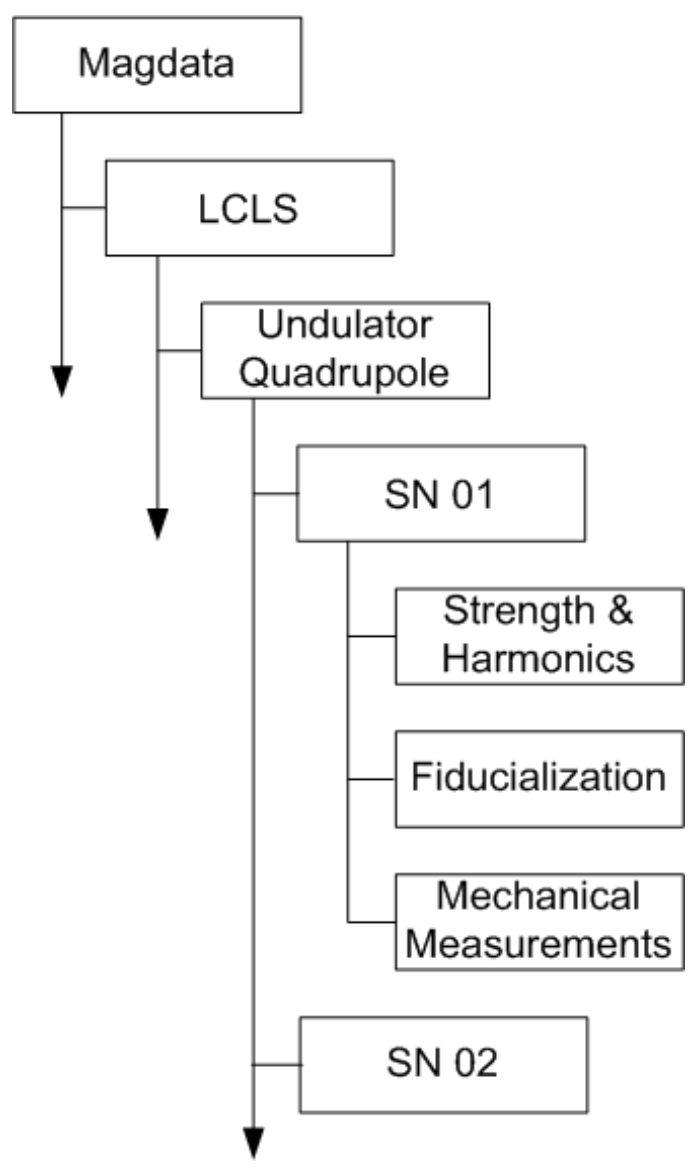

Figure 11: Directory structure in which the quadrupole fiducialization data is placed.

\section{Measurement Results}

All raw data and analysis results will be available from the SLAC web site. The data will be stored in a directory structure as shown in figure 11. The top level directory is Magdata, followed by $L C L S$, followed by the magnet type Undulator Quadrupole. In the Undulator Quadrupole directory, there is a folder for each quadrupole named by the serial number. Within each serial number folder, the Strength $\mathscr{E}$ Harmonics, Fiducialization, and Mechanical Measurements folders are created. Each contains all the relevant measurements. All data files will be text files. There will be several types of data files with different formats depending on the measurement.

All fiducialization data will be put in the Fiducialization folder. The data comes from several sources. Calibration data for the four wire position detectors will be placed in a file. The output of the program which automatically aligns the quadrupole axis to the wire will be placed in a file. All optical alignment measurements will be entered into a computerized form with the measurements written to a data file. CMM data will be written to a data file. Finally, the analysis program will summarize all of the data contained in the input 
files and give the offsets from the ideal beam axis to the quadrupole tooling balls.

An illustration of the various files required and their contents is given below. The file names are only suggestive. The names of the contents follow the conventions used in this note. In practice, redundant measurements are made which will be included in the files in addition to the minimal information given below.

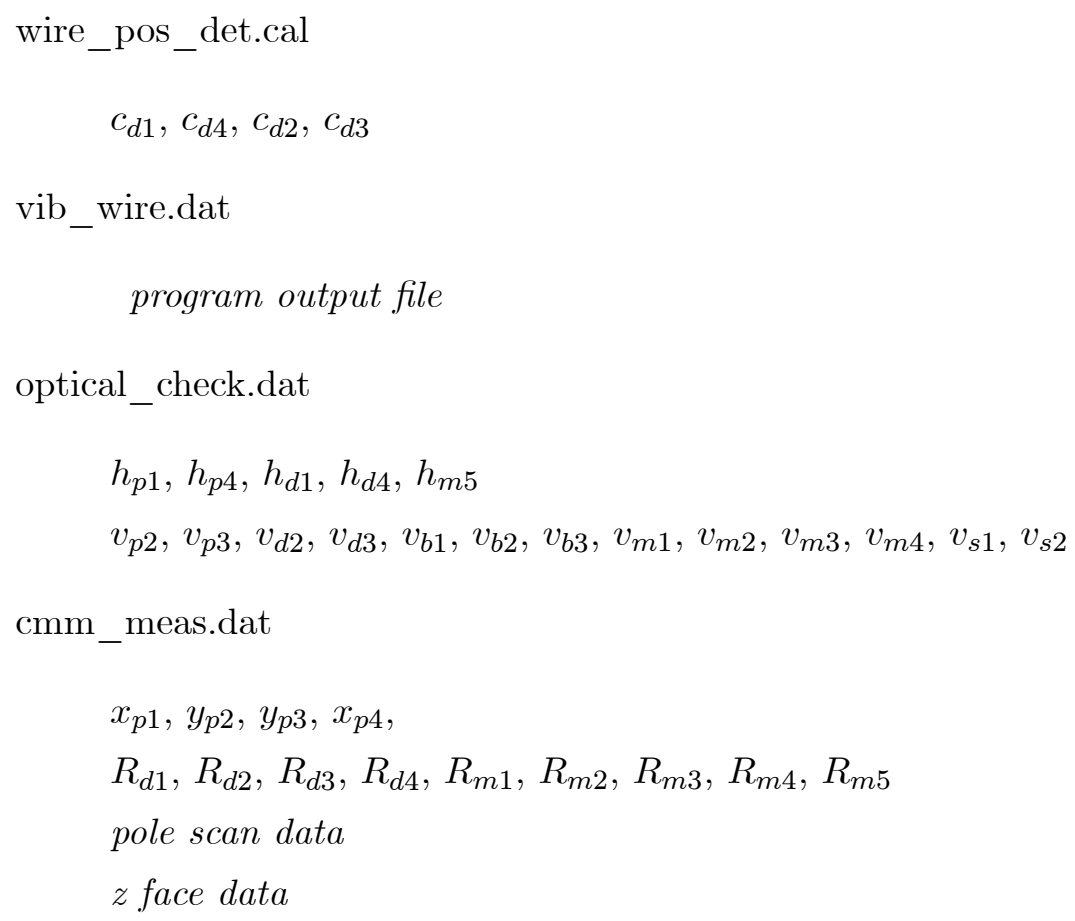

fiducialization_results.dat

$x_{m}, y_{m}, z_{m}$, roll $_{m}$, pitch $_{m}$, yaw $_{m}$ 